COMPUTATIONAL RESEARCH PROGRESS IN APPLIED SCIENCE \& ENGINEERING (CRPASE)
CRPASE: TRANSACTIONS OF

\title{
Provide A Method To Optimize the Reduction of Energy Consumption in Wireless Sensor Networks Using the Cuckoo Algorithm
}

\author{
Reza Molaee Fard* \\ Department Of Computer Engineering - Dezful Branch of Azad University, Khuzestan, Iran
}

\begin{tabular}{ll}
\hline Keywords & Abstract \\
\cline { 2 - 3 } $\begin{array}{l}\text { Cuckoo Algorithm, } \\
\text { Wireless Sensor Network, }\end{array}$ & $\begin{array}{l}\text { Given the high importance of wireless sensor networks in various applications, it is } \\
\text { necessary to examine the important challenges of these networks. The challenges of these } \\
\text { networks are the short life of the nodes, which leads to shortening the life of the network } \\
\text { Energy Reduction. }\end{array}$ \\
$\begin{array}{l}\text { and ultimately disupting the network. One of the ways to increase efficiency and reduce } \\
\text { energy consumption in the network is to use clustering methods. In this research, using } \\
\text { clustering methods and selection of efficient clusters by DEEC algorithm and optimization } \\
\text { of this algorithm using cuckoo algorithm. The results of the study showed an improvement } \\
\text { in reducing energy consumption in this type of network, so that the energy remaining in the } \\
\text { network after the simulation in routing with the new protocol is about 13\% more than TTDD } \\
\text { routing about } 11 \% \text { more than routing with APTEEN protocol. If we consider the life of the } \\
\text { network when the first node in the network is turned off, in the new protocol, the first node } \\
\text { is turned off } 7 \text { seconds later than the TTDD protocol and 13 seconds later than the APTEEN } \\
\text { protocol. This means that, on average, the lifespan of the network has been increased by } \\
\text { about } 10 \% \text { with the new protocol. }\end{array}$
\end{tabular}

\section{Introduction}

Increasing advances in the construction of integrated circuits as well as the development of wireless communications have led to the development of micro sensors that form the core of a wireless sensor network. In other words, a sensor network consists of nodes that are densely distributed in the environment and work together to perform a common task. Today, wireless sensor networks have special applications in various fields of military, health, habitat monitoring, industrial process monitoring and other fields. The combination of constraints and the way nodes are deployed in such networks doubles the need for energy-conscious designs at all network layers for the field. For example, in the network layer, when designing routing algorithms, the energy consumption limit parameter should be considered so that the proposed algorithm increases the life of the network and reduces energy consumption. Therefore, many challenges in sensor networks such as storage resources, computing capabilities, communication bandwidth and most importantly energy resources, in the last decade have created extensive research in this area. One of the suitable techniques to increase the lifespan of wireless sensor networks is the use of hierarchical routing.in this type of routing, nodes are placed in separate groups called clusters, and a cluster is selected for each cluster. Member nodes send their data to the header. The header receives and aggregates the data and sends it as a single step or in several steps to the base station called the well. Due to their low cost and easy communication, wireless sensor networks are used in many applications today for monitoring activities in different environments. The sensors in these networks use a limited power supply, after which, due to the non-renewable nature of these sources, the life of the network ends. To further use and extend the life of this type of network, researchers are always looking for a method. There are ways to reduce energy consumption in wireless sensor networks. Most of the energy consumed by these networks is spent on data

\footnotetext{
* Corresponding Author: Reza Molaee Fard

E-mail address: Rezamolae4@gmail.com
}

Received: 13 April 2021; Revised: 20 May 2021; Accepted: 26 May 2021

https://doi.org/10.52547/crpase.7.2.2336

Please cite this article as: R. Molaee Fard, Provide A Method To Optimize the Reduction of Energy Consumption in Wireless Sensor Networks Using the Cuckoo Algorithm, Computational Research Progress in Applied Science \& Engineering, CRPASE: Transactions of Electrical, Electronic and Computer Engineering 7 (2021) 1-7, Article ID: 2336. 
transmission. Sensor node clustering methods are one of the best methods that can significantly increase network life. Sensor nodes always have limited energy. Therefore, the efficient use of sensor node energy can extend the life of sensor networks. All sensors receive data from a fixed database or transmit it to a fixed database. This fixed database is called the base station, which usually acts as a server for other networks. The most important challenge in sensor networks is the limited energy resources of the sensor nodes. Therefore, the energy in each node is considered as a top priority in designing routing protocols. Communication protocols play an essential role in the efficiency and longevity of wireless sensor networks, so the design of practical protocols in energy consumption for wireless sensor networks is a necessity, so that using them not only reduces the total energy consumption of the network, Rather, the energy consumption load is evenly distributed among the network nodes, thus increasing the life of the network. In the proposed protocols, clustering based protocols significantly save energy consumed by the network. In this type of protocol, the whole network is divided into several clusters and in each cluster, one node is selected as the header. The task of the headers is to collect the data sent from the nodes of that cluster, remove duplicate data, combine the data and send this data to the sink. In these protocols, selecting one node in each cluster as the header and combining the data is greatly effective in increasing the scalability and longevity of the network. In this paper, we use the cuckoo algorithm to try to optimize the clustering of sensor nodes and select the minimum communication headers, which reduces the total communication distances and effectively reduces energy consumption and network life.

\section{Releted Work}

Latanzi et al , In a 2020 paper, Proposed the IEEE 802.15.4 controlled interference wireless networks as a function of packet length to increase network life, reliability, and energy efficiency. In their paper, they conclude that the results of extensive experiments on a wide range of protocols for asynchronous access and low media power point to a compromise between energy consumption and interference resistance, and provide a comparative view of these protocols [1].

Wang et al, In their 2018 paper Examined the genetic algorithm for energy-efficient clustering and routing in wireless sensor networks. In their paper, the researchers used an energy-based clustering and routing approach based on a genetic algorithm called GECR. They added the optimal solution obtained in the previous network round to the initial population for the current round. In this way, they improved the search efficiency. In addition, they combined clustering and routing schemes on a single chromosome to calculate total energy consumption and presented the proportionality function directly based on total energy consumption, thus increasing network efficiency and longevity. The results of these researchers' research showed that GECR achieved the best load balance with the least variance in loads and eclipses under different scenarios [2].
Jay et al, In their 2018 paper Presented a way to optimize the routing protocol in the TEEN network. In this paper, in order to further stabilize the routing and spectrum, the researchers presented an advanced energy-sensitive and threshold-sensitive sensor network (A_TEEN), which is an optimized version of the TEEN protocol. The A_TEEN protocol enhances the cluster selection method compared to energy efficiency and extends the lifespan of the wireless sensor network. The A_TEEN protocol improves energy efficiency and longevity by working more in the same structures compared to the conventional TEEN protocol [3].

Al-Sherkawi et al, In their 2018 paper, Presented an incremental approach to reducing energy consumption in wireless sensor networks. The researchers did this by boosting the energy balance in clusters across all sensor nodes, which reduced energy during network communication. This method is developed based on the method of selecting the head. In addition, an optimized TDMA scheduler has been implemented. Finally, the development approach reflects the improvement in network life, number of headers, power consumption, and number of packets transmitted to BS over other related protocols [4].

In their 2021 paper, Singh and Sharma proposed a new way to reduce energy consumption in wireless sensor networks. In their paper, the researchers used nature-inspired algorithms as a convenient way to optimize energy consumption. The researchers believed that optimal network coverage not only minimized the limited power consumption of battery sensors but also reduced the measurement of additional information. In this paper, two algorithms are used for the proposed method. The first is the genetically enhanced hybrid algorithm and the Ant Binary Colony Algorithm (IGA-BACA) and the second is the Optimization Lion (LO). The simulation results confirm that LO offers better network coverage and LO convergence speed is faster than IGA-BACA. In addition, it is observed that optimal coverage is achieved in a smaller number of LO generations compared to IGA-BACA. This study helps researchers to discover applications in this field as well as beyond [5].

In 2021, Tom et al. presented a new algorithm for optimizing and managing energy consumption in wireless sensor networks. In this research, several methods have been proposed to increase the life of the network, one of which involves the establishment of relay nodes to transfer data from sensors to the base station. However, the limited number of relay nodes is a challenge that is often overlooked. In this paper, the problem of optimizing network life and the number of relay nodes in three-dimensional fields is investigated. A new algorithm called MOEA / D-LS is proposed with the aim of achieving a better deal between the two objectives. The algorithm is a combination of a multiobjective evolutionary algorithm based on specific local analysis and search to optimize the previous sub problems. The simulation results in the three-dimensional data set show that the proposed algorithm performs much better than the algorithms in all the measured criteria [6].

In their article in 2021, Pakdel and fotohi proposed a new method to reduce energy consumption in wireless sensor networks using the firefly algorithm. The researchers argued that in wireless sensor networks (WSNs), designing a stable, 
low-power routing protocol is a major challenge because successive changes in connections or failures destabilize the network topology. Therefore, choosing the right path in this type of network due to limited resources and their operating environment is one of the most important challenges of these networks. Therefore, the main purpose of these networks is to collect appropriate routing information about the environment around the network sensors while observing the energy consumption of the sensors. In this paper, a method using the firefly algorithm and using the four residual energy criteria, noise level, number of hop and distance is presented. The proposed EM-FIREFLY method is introduced, which selects the best cluster head with high attractiveness and based on fitness performance, and transfers data packets through this cluster head to the sink. The proposed method is evaluated with NS-2 simulator and compared with PSOalgorithm and optimal clustering methods. The evaluation results show the efficiency of EM-FIREFLY method in maximum relative load and network life criteria compared to other methods discussed in this paper [7].

Al-Khadiri and her colleagues in 2021 introduced a new clustering method to increase the efficiency of wireless sensor networks. In this paper, we propose a new proposed clustering method called the Low Least Adaptive Clustering Hierarchy (MW-LEACH). Cluster heads $(\mathrm{CHs})$ are selected depending on the excess energy and distances in the MWLEACH. With the proposed scheme, from the initial set, the nodes are selected through the excess energy around the density center, thus leading to the composition of the initial set of $\mathrm{CH}$ candidates. These candidates collect their members' data by transferring in several directions and transferring specific data to BS. This particular methodology has fewer side effects in terms of message and time, and is fast and guarantees a level of error tolerance. In Matlab simulations and evaluations during the experimental phase, the proposed method exceeds the conventional advanced protocols by relying on the criteria of power performance, packet delivery, power consumption, grid life (duration) and latencies [8].

In 2021, Buta Zhou et al. proposed a way to balance the energy load in wireless sensor networks using clustering techniques. The researchers proposed an improved soft-kmeans (IS-k-means) clustering algorithm to balance the power consumption of nodes in WSN networks. This method first uses the idea of summation by quick search and finding peak densities (CFSFDP) and kernel density estimation (KDE) to improve the selection of primary cluster centers of the software clustering algorithm. Then, the soft flexibility $\mathrm{k}$ means and redefines the nodes according to the probability of their membership in the cluster boundary to balance the number of nodes in each cluster. In addition, the concept of multiline heads is used to balance energy consumption in clusters. Extensive simulation results under different network scenarios show that for small-scale WSNs with single-hop transmission, the proposed algorithm can delay the first node death, half node death and the last node death by an average compared to algorithms Different clustering to achieve higher performance [9].

\section{Suggested Method}

In the proposed method, a new method is proposed to reduce energy consumption in wireless sensor networks. The proposed method is that we first measure the amount of energy consumed in each sensor node and the life of the network, after determining the amount of energy in each node, we first divide the nodes into separate clusters. Then we select a header for each cluster. To reduce energy consumption using the cuckoo algorithm, clusters that consume a lot of energy are identified and converted into improved clusters with less energy consumption.

In the proposed method, we first obtain the energy consumption pattern through the following equation.

$$
\begin{gathered}
E_{r}=\left(E_{\text {elec }} * i\right)+\left(E_{\text {amp }} * i * d^{2}\right), E_{r} \\
=\left(E_{\text {elec }} * i\right)
\end{gathered}
$$

Where $E_{r}$ ' is the energy consumed by the data sending node. $E_{\text {elec }}$ ' is the energy required to send and receive a bit of information that does not depend on distance. $E_{a m p}$ is the energy required to amplify the transmitted signal over the intended distance. $i$ is the length of the message. $d$ is the distance to the node receiving the information.

\subsection{Network Life}

The lifespan of a network is actually the amount of time that the energy of the first sensor node runs out, or in other words, the length of time that the first node fails and the information is not measured. Generally, this time is normalized by dividing by its maximum value so that the value is in the range $[0,1]$. Hence the lifetime of the network is defined using the following relation $[10,11]$.

$$
f_{2}=\operatorname{Life}(x)=\frac{\operatorname{Min}\left\{T_{\text {failure }, i}\right\}_{i=1, \ldots, k}}{T_{\max }}
$$

In the above relation, $\mathrm{k}$ is the number of sensor nodes, $\operatorname{Min}\left\{T_{\text {failure }, i}\right\}_{i=1, \ldots, k}$ the number of measurement periods before the end of the energy of a sensor node and $T_{\max }$ the maximum possible number It is for assessment courses.

\subsection{Clustering of Nodes}

The main task of sensor network nodes is to collect information from the environment in which it is located. The main task of sensor network nodes is to collect information from the environment in which it is located. As a result, schemes that shorten communication distances can reduce energy consumption in the grid and increase grid life. The ideal state in sensor networks is such that the energy of all nodes ends together [12],13]. As a result, to increase the life of the network, the load distribution of the network is uniform to minimize the time interval between the death of the first node and the death of the last node. To achieve this goal, several communication protocols have been proposed so far, among which clustering-based protocols significantly reduce the power consumption of the network. Grouping nodes in clusters widely results in scalability and generally 
achieves higher energy efficiency and longer lifespan in wireless sensor networks [13], 14].

\subsection{Select a Header With The DEEC Algorithm}

The DEEC algorithm uses the residual energy to determine the eclipse. To prevent all nodes from having universal knowledge of the grid, the DEEC estimated an ideal value for grid life, which is used to calculate the reference energy that each node should use in one round [15, $16]$.

\subsection{Eclipse Selection Algorithm Based on Residual Energy}

$N_{\mathrm{i}}$ is the number of cycles that ninety $S_{i}$ turns on and is used as the rotation period. In heterogeneous networks, to ensure that we have an average of $\mathrm{P}_{\mathrm{opt}} * \mathrm{~N}$ per cycle, every node $\mathrm{S}_{\mathrm{i}}(\mathrm{i}=1,2, \ldots, \mathrm{n})$ is eclipsed per $1 / p_{\text {oot }}$ cycle.In LEACH The rotation period of $N_{I}$ is the same for all nodes. This prevents distributed energy from being consumed and some of the less energetic nodes to die earlier than other nodes, but DEEC considers different rotation periods for the nodes based on the remaining energy of the nodes $E_{i}(r)$.

$P_{i} \frac{1}{N_{i}}$ Title The average probability that node $\mathrm{i}$ will be eclipsed in far $N_{\mathrm{i}}$ When the nodes have the same energy in each period, $P_{\text {opt }}$ is used instead of $\mathrm{Pi}$, which ensures that $* \mathrm{~N} \mathrm{P}_{\mathrm{opt}}$ is present and all nodes die at almost the same time. But if the nodes have different amounts of energy, the $P_{i}$ node with the most energy should be larger than the $\mathrm{P}_{\text {opt }}$ $\bar{E}(r)$ is the average energy of a grid, which is calculated as follows:

$$
\bar{E}(r)=\frac{1}{n} \sum_{i=1}^{N} E i(r)
$$

To calculate this value, each node must be aware of the residual energy of all network nodes. It is also possible to estimate the value of $\bar{E}(r)$ using the following formulas:

$$
P i=P o p t\left[1-\frac{\bar{E}(r)-E i(r)}{E(r)}\right]=P o p t \frac{E i(r)}{\bar{E}(r)}
$$

As a result, the number of winners in each round will be as follows:

$$
\sum_{i=1}^{\mathrm{n}} \mathrm{Pi}=\sum_{\mathrm{i}=1}^{\mathrm{n}} \operatorname{Popt} \frac{E i(r)}{\bar{E}(r)}=N P o p t
$$

This is the optimal number of euphemisms we should have. We define a probability threshold that each $S_{i}$ node uses to determine whether it can be eclipsed during this period. The threshold is defined as follows:

$$
\mathrm{T}(\mathrm{Si})=\frac{P i}{1-\mathrm{Pi}\left(\operatorname{rmod}\left(\frac{1}{\mathrm{Pi}}\right)\right)} \quad \mathrm{Si} \in \mathrm{G}
$$

G Are a set of nodes that are eligible for eclipse at $r$. If the $S_{i}$ node is not encapsulated in the current $N_{i}$, it is added to set $G$.In each round $r$, when ninety $S_{i}$ has the condition of eclipse, it chooses a random number between 0 and 1 and compares the value produced with the threshold $\mathrm{T}\left(\mathrm{S}_{\mathrm{i}}\right)$. The value of $\mathrm{N}_{\mathrm{i}}$ in each round is the inverse of $\mathrm{S}_{\mathrm{I}}$, which is calculated based on the residual energy of ninety $S_{I}$ in round r:

$$
N i=\frac{1}{P i}=\frac{\bar{E}(r)}{\text { Popt } * E i(r)}=N o p t \frac{\bar{E}(r)}{E i(r)}
$$

Which is $N o p t=\frac{1}{\text { Popt }}$. This equation shows that $\mathrm{N}_{\mathrm{i}}$ is not the same for all nodes, and nodes with more residual energy shine at longer cycles than less energetic nodes.

\subsection{Dealing with Homogeneous Nodes}

$\mathrm{P}_{\text {opt }}$ is the reference value used to calculate $\mathrm{P}_{\mathrm{i}}$, Ni rotation period, and $\mathrm{T}\left(\mathrm{s}_{\mathrm{i}}\right)$ threshold for each node. In homogeneous networks, all nodes have the same initial energy, and therefore $\mathrm{P}_{\mathrm{opt}}$ is the same for all nodes, but in heterogeneous networks, this value is different for each node and is proportional to its initial energy. In heterogeneous networks, different $\mathrm{P}_{\mathrm{opt}}$ are considered for normal and advanced nodes:

$$
\begin{gathered}
\text { Padv }=\frac{\text { Popt }}{1+a m} \\
\text { Pnrm }=\frac{\text { Popt }(1+a)}{1+a m}
\end{gathered}
$$

As a result, $\mathrm{P}_{\mathrm{i}}$ will change as follows:

$$
\begin{aligned}
P i & =\frac{P o p t * E i(r)}{(1+a m) \bar{E}(r)} \quad \text { normal node }(8) \\
P i & =\frac{P o p t *(1+a)}{(1+a m) \bar{E}(r)} \quad \text { advance node }(9)
\end{aligned}
$$

To determine the threshold using Formula 9, it is observed that the threshold depends directly on the residual energy and the initial energy, and that nodes that have more energy have a better chance of rejoicing than nodes that have less energy, and this It causes a balanced distribution of energy in the network.

This model can be easily generalized to heterogeneous multilayer networks, in which case the weight probability should be used, which is calculated as follows:

$$
P(S i)=\frac{P o p t * N *(1+a i)}{N+\sum_{i=1}^{n} a i}
$$




\subsection{Estimate the Average Energy}

To find the average network energy, each node must have global knowledge of the whole network, this action imposes a lot of overhead on the network, to prevent this, the average energy in the network is estimated.

$$
\bar{E}(r)=\frac{1}{N} \operatorname{Etotal}\left(1-\frac{r}{R}\right)
$$

Where $\mathrm{R}$ is the total number of cycles during the network life. This means that all nodes consume the same amount of energy per cycle. The DEEC algorithm determines the $N_{i}$ rotation period for each node in terms of the residual energy of the nodes and controls the energy consumption in each cycle. As a result, the actual energy of each node is affected by the reference energy $\bar{E}(r)$. This algorithm thus ensures that almost all nodes die at the same time. In fact, the most important goal of the DEEC algorithm is to control energy consumption based on an adaptive approach.

To calculate $\bar{E}(r)$, we must first know the value of R. As mentioned earlier, $\mathrm{R}$ is the total number of network cycles until the death of all nodes. $E_{\text {rount }}$ is the energy consumed per network cycle that can be used to estimate $\mathrm{R}$ as follows:

$$
\mathrm{R}=\frac{\text { Etotal }}{\text { Eround }}
$$

In this protocol for $\mathrm{L}$ bit transmission, its energy consumption is calculated as follows:

$$
\begin{aligned}
& L * \text { Eelec }+L * \text { eefs } * d^{2} \quad d<d 0 \\
& \operatorname{Etx}(L, d)=
\end{aligned}
$$

In this equation, $E_{\text {elec }}$ uses the energy consumed to receive or send 1 bit of data, $\varepsilon e f s * d^{2}$ and $\varepsilon m p * d^{4}$, the energy of the amplifier, which is set according to the distance and the transmission model (free environment or multiple environment).

Suppose $\mathrm{N}$ nods are randomly distributed in the $\mathrm{M}^{*} \mathrm{M}$ environment and the central station is in the center of this square. Each non-spinning node sends an $\mathrm{L}$ bit to the spinning node in each round. The total energy consumption in a grid cycle is calculated according to the following formula:

$$
\begin{array}{r}
\text { Eround }=\mathrm{L}(2 * \mathrm{~N} * \text { Eelec }+\mathrm{N} * \text { Eda }+\mathrm{K} * \varepsilon \mathrm{mp} \\
\left.* \mathrm{~d}^{4} \text { toBS }+\mathrm{N} * \varepsilon \text { efs } * \mathrm{~d}^{2} \text { toCH }\right)
\end{array}
$$

In this equation $\mathrm{K}$ is the number of clusters, $E_{d a}$ is the energy required to aggregate the data in the cluster, $d_{t o B S}$ is the cluster distance to the main station and $d_{t o C H}$ is the distance of the node members of the cluster to their cluster. Suppose the nodes are evenly distributed:

$$
d t o C H=\frac{M}{\sqrt{2 \pi k}}
$$

$$
d t o B S=0.76 \frac{M}{2}
$$

If we derive $E_{\text {round }}$ from $\mathrm{k}$ and set it to zero, we get the optimal number of headers:

$$
K o p t=\frac{\sqrt{n}}{\sqrt{2 \pi}} \sqrt{\frac{\varepsilon f s * M}{\varepsilon m p * d^{2} t o B S}}
$$

Using this formula, $E_{\text {round }}$ can be calculated and as a result, $\mathrm{R}$ is calculated. In the DEEC algorithm, the base station calculates the total $\mathrm{E}_{\text {total }}$ energy and the estimated value of $\mathrm{R}$ and notifies all nodes. At the beginning of each cycle, each $S_{i}$ node receives this information, calculates the probability average $\mathrm{P}_{\mathrm{i}}$, the average network energy, and the threshold, and the protocol routine continues.

\section{Optimization of Node Energy Using Cuckoo Algorithm}

In the next step, we will optimize the node energy using the cuckoo meta-heuristic algorithm. The cuckoo algorithm is one of the newest and most powerful evolutionary optimization methods. This algorithm uses the lifestyle of a bird named Face. This algorithm starts with an initial population. This population of cuckoos has a number of eggs that they will lay in the nest of a number of host birds. Some of these eggs are more similar to the eggs of the host bird, more likely to be the eggs of the host bird, more likely to grow into an adult cuckoo. Other eggs identified by the host bird are destroyed. The amount of eggs grown indicates the suitability of the nests in that area. The more eggs that can live and survive in an area, the more profit will be made to that area; Therefore, the situation in which the largest number of eggs are saved will be a parameter that the cuckoos intend to optimize [17], [18] , [19].

- To solve an optimization problem, it is necessary to form the values of the problem variables in the form of an array.

- In the cuckoo algorithm, the array is called Habitat.

- $\quad$ in the next $N_{v a r}$ optimization problem, a Habitat will be an array var $1 \times_{\text {var }}$ that represents the current living position of the cuckoos. This array is defined as follows.

$$
\text { Habitat }=\left[x_{1}, x_{2}, x_{3}, \ldots, \ldots, x_{\text {Nvar }}\right]
$$

- The appropriateness of the current Habitat is obtained by evaluating $\left(f_{p}\right)$ in the Habitat, therefore:

$$
\begin{aligned}
& \text { Profit }=f_{p} \text { habitat } \\
& \qquad \quad f_{p}\left(x_{1}, x_{2}, x_{3}, \ldots, \ldots, x_{N v a r}\right)
\end{aligned}
$$

- As can be seen, the cuckoo algorithm is an algorithm that maximizes the profit function. 
- To use the cuckoo algorithm to solve the minimization problems, it is enough to multiply a negative sign in the cost function.

- To start the optimization algorithm, we generate a Habitat matrix with size $N_{\text {pop }} \times N_{\text {var }}$.

- A number of random eggs are then assigned to each of these habitats.

- In nature, each cuckoo lays between 5 and 20 eggs. These numbers are used as the upper and lower limits of each cuckoo egg allocation in different iterations.

$$
\begin{gathered}
E L R=a \times \frac{\text { Number of current cuckoos eggs }}{\text { Total number of eggs }} \\
\times\left(V_{\text {a }} \text { eri }-V a r_{\text {low }}\right)
\end{gathered}
$$

\subsection{Applications of The Cuckoo Algorithm}

- Evolutionary algorithms can be applied to any problem that can be used as performance optimization problems.

- It is an algorithm for solving continuous and nonlinear optimization problems.

- This algorithm is able to converge to a close approximation of the optimal global solution in a small number of iterations.

- Its high performance and ability to solve optimization problems.

- Resistant to dynamic changes.

- The cuckoo algorithm can be combined with more traditional optimization techniques

- This algorithm has high speed and accuracy in converging to the optimal solution of standard objective functions.

- In cases where the objective function has a large number of local optimal answers, this algorithm is able to converge to a close approximation of the global optimal answer in a small number of iterations.

- This algorithm is one of the newest and most powerful evolutionary optimization methods that has more ability to find global optimal points.

- Solves problems that have no solution: The advantages of evolutionary algorithms include the ability to solve problems for which there is no human expertise. Even if human expertise should be used when needed and available. It is often less difficult for automated problem-solving procedures.

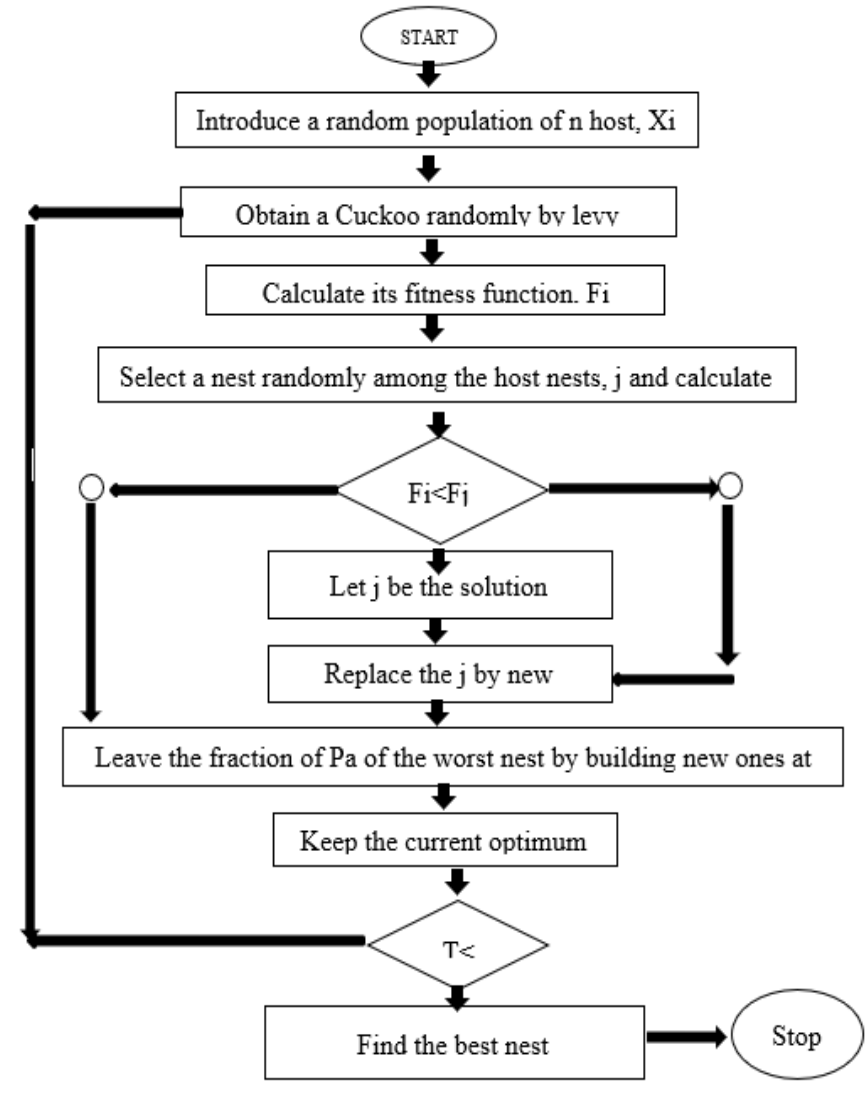

Figure 1. shows the framework of the cuckoo algorithm

At the end of optimizing the reduction of energy consumption in sensor networks using the cuckoo algorithm, we see a significant reduction in energy consumption in sensor networks. The results of improving the performance of the proposed method can be seen in the evaluation of the proposed method.

\section{Evaluate The Proposed Method}

To evaluate the proposed method, a comparison was made between the proposed method algorithm and the two algorithms TTDD and APTEEN in terms of comparing the network life and energy consumption, which resulted in improved sensor networks in terms of energy consumption. You can see the extent of this improvement in the charts below.

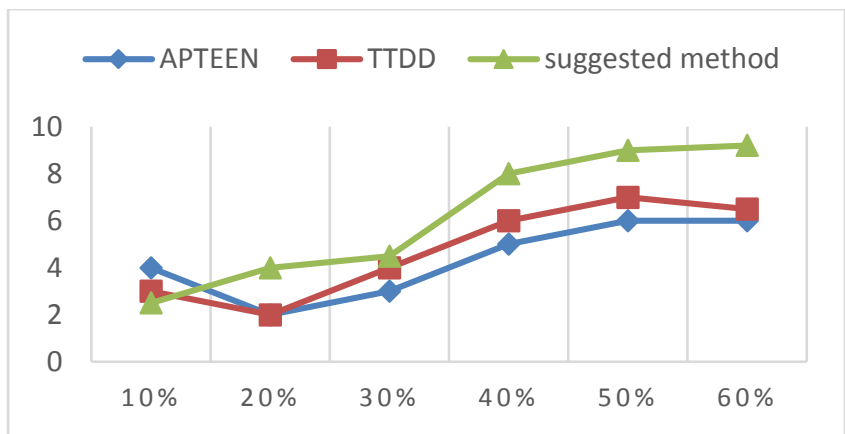

Figure 2. Comparison of network life with the proposed method and comparison with two protocols TTDD and APTEEN 


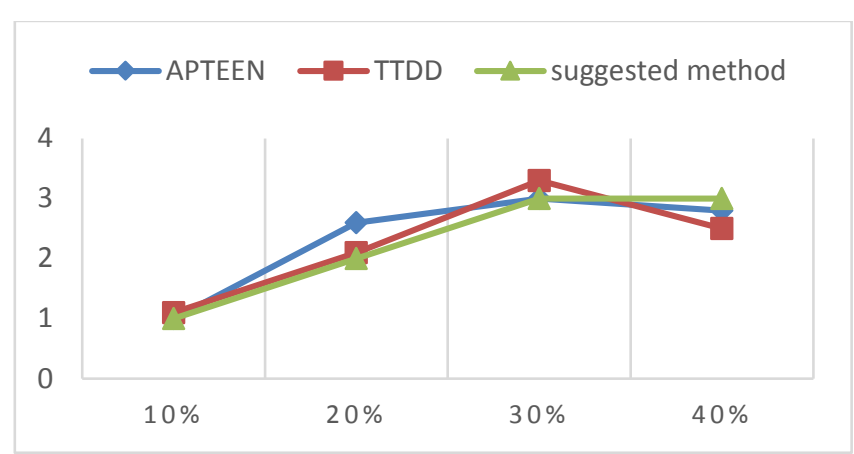

Figure 3. Comparison of grid energy consumption with the proposed method and comparison with two protocols TTDD and APTEEN

\section{Conclusion}

In wireless sensor networks, clustering-based routing protocols work best by dividing neighboring nodes into separate clusters and selecting local clusters to combine and send information from each cluster to the base station and try to consume power by the network nodes. In terms of increasing longevity and maintaining network coverage compared to other routing methods. Different features of wireless sensor networks such as limited power supply, density, low bandwidth, small memory sizes and limited energy make the network vulnerable. The limited battery life of these networks has made energy consumption in wireless networks one of the main challenges. Different methods have been proposed to reduce energy consumption in wireless sensor networks. Among these, clustering routing algorithms have made the most effort. In this research, with the help of clustering algorithm based on DEEC algorithm and optimization of these algorithms using cuckoo algorithm, it was tried to reduce energy consumption in sensor networks. In clustering sensor networks, one of the important indicators that is effective in the amount of data received in the well, increasing network life and reducing waste energy, is the selection of the appropriate head. The results of the study showed an improvement in reducing energy consumption in this type of network, so that the energy remaining in the network after the simulation in routing with the new protocol is about $13 \%$ more than TTDD routing about $11 \%$ more than routing with APTEEN protocol. If we consider the life of the network when the first node in the network is turned off, in the new protocol, the first node is turned off 7 seconds later than the TTDD protocol and 13 seconds later than the APTEEN protocol. This means that the average lifespan of the network has been increased by about $10 \%$ with the new protocol.

\section{References}

[1] E. Lattanzi, P. Capellacci, V. Freschi, Experimental evaluation of the impact of packet length on wireless sensor networks subject to interference, Computer Networks 167 (2020) 106986.

[2] T. Wang, G. Zhang, X. Yang, A. Vajdi, Genetic algorithm for energy-efficient clustering and routing in wireless sensor networks, Journal of Systems and Software 146 (2018) 196214

[3] Y. Ge, S. Wang, J. Ma, Optimization on TEEN routing protocol in cognitive wireless sensor network, EURASIP
Journal on Wireless Communications and Networking (2018) $1-9$.

[4] M. Elshrkawey, SM. Elsherif, ME. Wahed, An enhancement approach for reducing the energy consumption in wireless sensor networks, Journal of King Saud University-Computer and Information Sciences 30 (2018) 259-267.

[5] J. Wu, K. Hu, Y. Cheng, H. Zhu, X. Shao, Y. Wang, Datadriven remaining useful life prediction via multiple sensor signals and deep long short-term memory neural network." ISA transactions 97 (2020) 241-250.

[6] NT. Tam, TH. Hung, HTT. Binh, A decomposition-based multi-objective optimization approach for balancing the energy consumption of wireless sensor networks, Applied Soft Computing (2021) 107365.

[7] H. Pakdel, R. Fotohi, A firefly algorithm for power management in wireless sensor networks (WSNs), The Journal of Supercomputing (2021) 1-22.

[8] S. El Khediri, RU. Khan, N. Nasri, Energy efficient adaptive clustering hierarchy approach for wireless sensor networks, International Journal of Electronics 108 (2021) 67-86.

[9] B. Zhu, E. Bedeer, HH. Nguyen, Improved Soft-k-Means Clustering Algorithm for Balancing Energy Consumption in Wireless Sensor Networks, IEEE INTERNET OF THINGS JOURNAL 8 (2021).

[10] J. Van Dijk. The network society. Sage, 2020.

[11] U. Abdillah, S. Suyanto, Clustering Nodes and Discretizing Movement to Increase the Effectiveness of HEFA for a CVRP, International Journal of Advanced Computer Science and Applications (IJACSA) 11 (2020) 774-779.

[12] F. Zhang, S. Qi, Q. Liu, M. Mao, A. Zeng, Alleviating the data sparsity problem of recommender systems by clustering nodes in bipartite networks, Expert Systems with Applications 149 (2020) 113346.

[13] W. Osamy, A. Salim, AM. Khedr, An information entropy based-clustering algorithm for heterogeneous wireless sensor networks, Wireless Networks 26 (2020) 1869-1886.

[14] X. Liu, R. Zhu, A. Anjum, J. Wang, H. Zhang, Intelligent data fusion algorithm based on hybrid delay-aware adaptive clustering in wireless sensor networks, Future Generation Computer Systems 104 (2020) 1-14.

[15] S. Vançin, E. Erdem, Threshold balanced sampled DEEC model for heterogeneous wireless sensor networks, Wireless Communications and Mobile Computing (2018).

[16] F. Jibreel, Improved Enhanced Distributed Energy Efficient Clustering (iE-DEEC) scheme for heterogeneous wireless sensor network, International Journal of Engineering Research and Advanced Technology (IJERAT) 5 (2019) 6-11.

[17] HR. Boveiri, An enhanced cuckoo optimization algorithm for task graph scheduling in cluster-computing systems, Soft Computing 24 (2020) 10075-10093.

[18] X. Cai, Y. Niu, S. Geng, J. Zhang, Z. Cui, An under-sampled software defect prediction method based on hybrid multiobjective cuckoo search, Concurrency and Computation: Practice and Experience 32 (2020) 5478.

[19] M .Inci, A. Caliskan, Performance enhancement of energy extraction capability for fuel cell implementations with improved Cuckoo search algorithm, International Journal of Hydrogen Energy 45 (2020) 11309-11320. 